\title{
A SINGULAR INTEGRAL APPROACH TO A TWO PHASE FREE BOUNDARY PROBLEM
}

\author{
SIMON BORTZ AND STEVE HOFMANN
}

\begin{abstract}
We present an alternative proof of a result of Kenig and Toro [KT4], which states that if $\Omega \subset \mathbb{R}^{n+1}$ is a two sided NTA domain, with Ahlfors-David regular boundary, and the $\log$ of the Poisson kernel associated to $\Omega$ as well as the $\log$ of the Poisson kernel associated to $\Omega_{\mathrm{ext}}$ are in VMO, then the outer unit normal $v$ is in VMO. Our proof exploits the usual jump relation formula for the non-tangential limit of the gradient of the single layer potential. We are also able to relax the assumptions of Kenig and Toro in the case that the pole for the Poisson kernel is finite: in this case, we assume only that $\partial \Omega$ is uniformly rectifiable, and that $\partial \Omega$ coincides with the measure theoretic boundary of $\Omega$ a.e. with respect to Hausdorff $H^{n}$ measure.
\end{abstract}

\section{INTRODUCTION}

The study of free boundary problems for Poisson kernels, in which the regularity of the Poisson kernel $k$ associated to a domain $\Omega$, is shown to imply regularity of its boundary $\partial \Omega$, began with the work of Alt and Caffarelli [AC]. In $[\mathrm{AC}]$ it is shown that in the presence of sufficient Reifenberg flatness, plus Ahlfors-David regularity of the boundary, $\log k \in C^{\alpha} \Longrightarrow v \in C^{\beta}$, where $v$ is the unit normal to $\partial \Omega$. In [J], Jerison showed that $\beta$ can be taken to be equal to $\alpha$. In the same work, Jerison studied the regularity of the unit normal under the assumption that $\log k$ was continuous and that $\partial \Omega$ was Lipschitz. The converse to the Alt-Caffarelli-Jerison $C^{\alpha}$ result is a classical theorem of Kellog [Kel].

Kenig and Toro investigated what may be considered the 'end point' case of the results in $[\mathrm{AC}]$, again under the assumption of Reifenberg flatness and AhlforsDavid regularity. In [KT1] and [KT3], they showed that given those background hypotheses, one obtains

$$
\log k \in V M O(d \sigma) \Longleftrightarrow v \in V M O(d \sigma)
$$

(more precisely, for the direction " $\Longrightarrow$ ", they show that $v \in V M O_{l o c}(d \sigma)$; we shall return to this point below), where for a domain $\Omega \subset \mathbb{R}^{n+1}$, we let $\sigma:=\left.H^{n}\right|_{\partial \Omega}$ denote the surface measure on $\partial \Omega$, where as usual $H^{n}$ denotes $n$-dimensional Hausdorff measure. It is well known that in the absence of Reifenberg flatness, $\log k \in$ $V M O(d \sigma)$ need not imply that $v \in V M O(d \sigma)$ : the counterexample is the double

Date: August 6, 2014

2010 Mathematics Subject Classification. 42B20, 31B05, 31B25, 35J08, $35 \mathrm{~J} 25$.

Key words and phrases. Singular integrals, layer potentials, free boundary problems, Poisson kernels, VMO.

The authors were supported by NSF grant DMS-1361701. 
cone (see $[\mathrm{AC}]$ and $[\mathrm{KP}]$ ). With this in mind one may ask under what other hypotheses do we have $\log k \in V M O(d \sigma) \Longrightarrow v \in V M O(d \sigma)$, and when do we have $\log k \in V M O(d \sigma) \Longleftrightarrow v \in V M O(d \sigma)$ ?

The second author, along with M. Mitrea and M. Taylor [HMT], showed that if $\Omega$ is 2-sided NTA, with Ahlfors-David regular ("ADR") boundary, then $v \in$ $V M O(d \sigma)$ implies vanishing Reifenberg flatness, and hence that $\log k \in V M O(d \sigma)$, by the result of [KT1]. Thus, it seems reasonable to conjecture that in the setting of a 2-sided NTA domain with ADR boundary, $\log k \in V M O(d \sigma) \Longrightarrow v \in$ $V M O(d \sigma)$. Indeed, this very question was posed in [KT4]. In trying to answer this question (which remains open), the authors of the present work found an alternative approach, based on $L^{p}$ bounds and jump relations for layer potentials, to prove a two-phase version of the problem, which had previously been treated in [KT4].

In [KT4] Kenig and Toro established the following: suppose that $\Omega$ is a 2-sided chord-arc domain (i.e, a 2-sided NTA domain with ADR boundary), and that $k_{1}$ and $k_{2}$ are the Poisson kernels of $\Omega$ and $\Omega_{\text {ext }}$ respectively, with some fixed poles (either finite or infinite). If $\log k_{1}, \log k_{2} \in V M O(d \sigma)$ (or even $V M O_{l o c}$ ), then $v \in V M O_{l o c}(d \sigma)$. Their proof uses a blow-up argument which is quite natural given the nature of the problem. This blow-up argument, as well as our arguments here, require that one work with local versions of $V M O$; see Definition 1.25 below. In the present paper, we will use $L^{p}$ bounds and jump relations for the gradient of the single layer potential to show that if $\log k_{1}, \log k_{2} \in V M O_{l o c}(d \sigma)$, then $v \in$ $V M O_{l o c}(d \sigma)$. Our approach has the additional modest virtue that it allows us to weaken the 2-sided NTA condition in a non-trivial way, in the case that our Poisson kernels have finite poles. More precisely, our main result is the following. The terminology used in the theorem and throughout this introduction will be defined in the sequel.

Theorem 1.1. Let $\Omega$ and $\Omega_{\mathrm{ext}}$ be connected domains in $\mathbb{R}^{n+1}$, whose common boundary $\partial \Omega=\partial \Omega_{\text {ext }}$ is uniformly rectifiable, and whose measure theoretic boundary $\partial_{*} \Omega$ satisfies $\sigma\left(\partial \Omega \backslash \partial_{*} \Omega\right)=0 .{ }^{1}$ Suppose that there is some fixed pair of points $X_{1} \in \Omega$ and $X_{2} \in \Omega_{\mathrm{ext}}$, such that $\log k_{1}^{X_{1}}, \log k_{2}^{X_{2}} \in V M O_{\text {loc }}(d \sigma)$, where $k_{1}$ and $k_{2}$ are the Poisson kernels for $\Omega$ and $\Omega_{\mathrm{ext}}$ respectively. Then $\nu \in V M O_{l o c}(d \sigma)$.

Thus, uniform rectifiability of the boundary, along with the hypothesis that the measure theoretic boundary has full measure, replace the stronger 2-sided chord arc condition ${ }^{2}$, at least in the case that our Poisson kernels have finite poles. On the other hand, in the case that the Poisson kernel has pole at infinity, we impose an NTA hypothesis, as in [KT4]. Our second result is as follows.

\section{Theorem 1.2.}

(1) Suppose that $\Omega$ is an unbounded chord arc domain with $\Omega_{\text {ext }}$ connected, that $\log k_{1} \in V M O(d \sigma)$, where $k_{1}$ is the Poisson kernel for $\Omega$ with pole at

\footnotetext{
${ }^{1}$ Following [HMT], we use the term "UR domain", to refer to a domain whose boundary enjoys these properties; see Definition 1.14 below.

${ }^{2}$ It is known that chord arc domains have uniformly rectifiable boundaries [DJ], and in fact, the chord arc condition is strictly stronger.
} 
infinity, and that $\log k_{2}^{X_{2}} \in V M O_{l o c}(d \sigma)$, where $k_{2}^{X_{2}}$ is the Poisson kernel for $\Omega_{\text {ext }}$ with pole $X_{2} \in \Omega_{\text {ext }}$. Then $v \in V M O_{\text {loc }}(d \sigma)$.

(2) Suppose $\Omega$ is an unbounded 2-sided chord-arc domain, with unbounded complement, and that $\log k_{1}, \log k_{2} \in V M O(d \sigma)$, where $k_{1}$ is the Poisson kernel for $\Omega$ with pole at infinity, and $k_{2}$ is the Poisson kernel for $\Omega_{\mathrm{ext}}$ with pole at infinity. Then $v \in V M O(d \sigma)$.

As an immediate consequence, we obtain the following, as in [KT4].

Corollary 1.3. Suppose that $\Omega$ is a 2-sided chord-arc domain, and let $k_{1}, k_{2}$ be the Poisson kernels for $\Omega$ and for $\Omega_{\text {ext }}$, respectively, with fixed poles which may either be finite or infinite. Suppose also that $\log k_{1} \in C^{\alpha}$, and that $\log k_{2} \in V M O_{l o c}(d \sigma)$. Then $\partial \Omega$ is locally the graph of a $C^{1, \alpha}$ function.

Let us indicate the proof the the Corollary in the case that $\partial \Omega$ is bounded (then $V M O_{l o c}=V M O$ in this case). Observe that we may invoke Theorem 1.1 or Theorem 1.2 to deduce that $v \in V M O$. Consequently, by the aforementioned result of [HMT], we find that $\Omega$ is a vanishing Reifenberg domain. Thus, by the AltCaffarelli-Jerison theorem ([AC], [J]), $v \in C^{\alpha}(\partial \Omega)$. If $\partial \Omega$ is unbounded, we note that the results of $[\mathrm{AC}]$ and $[\mathrm{J}]$ are local in nature, and furthermore observe that the result in [HMT] may be localized as well, with essentially the same proof.

Throughout the present paper $\Omega$ will be a connected open (proper) subset of $\mathbb{R}^{n+1}$. We begin by setting notation, and recalling some definitions.

\subsection{Notation and Definitions.}

- We use the letters $c, C$ to denote harmless positive constants, not necessarily the same at each occurrence, which depend only on dimension and the constants appearing in the hypotheses of the theorems (which we refer to as the "allowable parameters"). We shall also sometimes write $a \lesssim b$ and $a \approx b$ to mean, respectively, that $a \leq C b$ and $0<c \leq a / b \leq C$, where the constants $c$ and $C$ are as above, unless explicitly noted to the contrary.

- Unless otherwise explicitly stated we will use lower case letters $x, y, z$, etc., to denote points on $\partial \Omega$, and capital letters $X, Y, Z$, etc., to denote generic points in $\mathbb{R}^{n+1}$ (especially those in $\Omega$ and $\Omega_{\text {ext }}$ ).

- The open $(n+1)$-dimensional Euclidean ball of radius $r$ will be denoted $B(x, r)$ when the center $x$ lies on $\partial \Omega$, or $B(X, r)$ when the center $X \in(\partial \Omega)^{c}$. A "surface ball" is denoted $\Delta(x, r):=B(x, r) \cap \partial \Omega$.

- Given a domain $\Omega$, for $X \in \mathbb{R}^{n+1}$, we set $\delta(X):=\operatorname{dist}(X, \partial \Omega)$.

- We let $H^{n}$ denote $n$-dimensional Hausdorff measure, and let $\sigma:=\left.H^{n}\right|_{E}$ denote the "surface measure" on a closed set $E \subset \mathbb{R}^{n+1}$ of co-dimension 1 .

- For a Borel set $A \subset \mathbb{R}^{n+1}$, we let $1_{A}$ denote the usual indicator function of $A$, i.e. $1_{A}(x)=1$ if $x \in A$, and $1_{A}(x)=0$ if $x \notin A$.

- For a Borel set $A \subset \mathbb{R}^{n+1}$, we let $\operatorname{int}(A)$ denote the interior of $A$.

- Given a Borel measure $\mu$, and a Borel set $A$, with positive and finite $\mu$ measure, we set $f_{A} f d \mu:=\mu(A)^{-1} \int_{A} f d \mu$. 
Definition 1.4. (ADR) (aka Ahlfors-David regular). We say that a set $E \subset \mathbb{R}^{n+1}$, of Hausdorff dimension $n$, is ADR if it is closed, and if there is some uniform constant $C$ such that

$$
\frac{1}{C} r^{n} \leq \sigma(\Delta(x, r)) \leq C r^{n}, \quad \forall r \in(0, \operatorname{diam}(E)), x \in E,
$$

where $\operatorname{diam}(E)$ may be infinite.

Definition 1.6. (Riesz transforms and the single layer potential) Let $E \subset \mathbb{R}^{n+1}$ be an $n$-dimensional ADR (hence closed) set with surface measure $\sigma$. We define the (vector valued) Riesz kernel as

$$
\mathcal{K}(x)=\tilde{c}_{n} \frac{x}{|x|^{n+1}}
$$

where $\tilde{c}_{n}$ is chosen so that $\mathcal{K}$ is the gradient of fundamental solution to the Laplacian. For a Borel measurable function $f$, we then define the Riesz transform

$$
\mathcal{R} f(X):=\mathcal{K} *(f \sigma)(X)=\int_{E} \mathcal{K}(X-y) f(y) d \sigma(y) \quad X \in \mathbb{R}^{n+1},
$$

as well as the truncated Riesz transforms

$$
\mathcal{R}_{\varepsilon} f(X):=\int_{E \cap\{|X-y|>\varepsilon\}} \mathcal{K}(X-y) f(y) d \sigma(y), \quad \varepsilon>0 .
$$

We define $\mathcal{S}$ the single layer potential for the Laplacian relative to $E$ to be

$$
\mathcal{S} f(X):=\int_{E} \mathcal{E}(X-y) f(y) d \sigma(y),
$$

where $\mathcal{E}(X)=c_{n}|X|^{1-n}$ is the (positive) fundamental solution to the Laplacian in $\mathbb{R}^{n+1}$. Notice that $\nabla \mathcal{S} f(X)=\mathcal{R} f(X)$ for $X \notin E$.

Definition 1.10. (UR) (aka uniformly rectifiable). There are numerous characterizations of UR sets (many of which remain valid in higher co-dimensions); we refer the interested reader to [DS1, DS2] for details. For our purposes, it is most useful to use the following definition. Let $E \subset \mathbb{R}^{n+1}$ be an $n$-dimensional ADR (hence closed) set with surface measure $\sigma$. Then $E$ is UR if and only if the Riesz transform operator is $L^{2}$ bounded with respect to surface measure, in the sense that

$$
\sup _{\varepsilon>0}\left\|\mathcal{R}_{\varepsilon} f\right\|_{L^{2}(E, \sigma)} \leq C\|f\|_{L^{2}(E, \sigma)} \text {. }
$$

See [DS1] for a proof of the fact that UR (defined in various other ways) implies (1.11). For the converse, see [MMV] in the case $n=1$, and [NToV] in general. For our purposes, we shall require only the first implication (UR implies (1.11)).

Remark 1.12. We note that the principal value $\mathcal{R} f(x)=\lim _{\varepsilon \rightarrow 0} \mathcal{R}_{\mathcal{E}} f(x)$ exists for a.e. $x \in E$, provided that $E$ is rectifiable, thus, in particular, if $E$ is UR (see, e.g., [M, Theorem 20.28]). Of course, the $L^{2}$ bound in (1.11) holds also for the principal value operator. Moreover, by standard Calderón-Zygmund theory, the $L^{2}$ bound self-improves to give $L^{p}$ bounds, $1<p<\infty$.

Definition 1.13. ("UR character"). Given a UR set $E \subset \mathbb{R}^{n+1}$, its "UR character" is just the constant $C$ in (1.11), along with the ADR constant; or equivalently, the quantitative bounds involved in any particular characterization of uniform rectifiability. 
Definition 1.14. (UR domain). Following the terminology in [HMT], we will say that a domain $\Omega$ is a UR domain if $\partial \Omega$ is UR, and if the measure theoretic boundary $\partial_{*} \Omega$ (see Definition 1.19 below) satisfies $\sigma\left(\partial \Omega \backslash \partial_{*} \Omega\right)=0$.

Definition 1.15. (Corkscrew condition). Following [JK], we say that a domain $\Omega \subset \mathbb{R}^{n+1}$ satisfies the "Corkscrew condition" if for some uniform constant $c>0$ and for every surface ball $\Delta:=\Delta(x, r)$, with $x \in \partial \Omega$ and $0<r<\operatorname{diam}(\partial \Omega)$, there is a ball $B\left(X_{\Delta}, c r\right) \subset B(x, r) \cap \Omega$. The point $X_{\Delta} \subset \Omega$ is called a "Corkscrew point" relative to $\Delta$. We note that we may allow $r<C \operatorname{diam}(\partial \Omega)$ for any fixed $C$, simply by adjusting the constant $c$.

Definition 1.16. (Harnack Chain condition). Again following [JK], we say that $\Omega$ satisfies the Harnack Chain condition if there is a uniform constant $C$ such that for every $\rho>0, \Lambda \geq 1$, and every pair of points $X, X^{\prime} \in \Omega$ with $\delta(X), \delta\left(X^{\prime}\right) \geq \rho$ and $\left|X-X^{\prime}\right|<\Lambda \rho$, there is a chain of open balls $B_{1}, \ldots, B_{N} \subset \Omega, N \leq C(\Lambda)$, with $X \in B_{1}, X^{\prime} \in B_{N}, B_{k} \cap B_{k+1} \neq \varnothing$ and $C^{-1} \operatorname{diam}\left(B_{k}\right) \leq \operatorname{dist}\left(B_{k}, \partial \Omega\right) \leq C \operatorname{diam}\left(B_{k}\right)$. The chain of balls is called a "Harnack Chain".

Definition 1.17. (NTA and 2-sided NTA). Again following [JK], we say that a domain $\Omega \subset \mathbb{R}^{n+1}$ is NTA ("Non-tangentially accessible") if it satisfies the Harnack Chain condition, and if both $\Omega$ and $\Omega_{\text {ext }}:=\mathbb{R}^{n+1} \backslash \bar{\Omega}$ satisfy the Corkscrew condition (for domains). If $\Omega$ and $\Omega_{\text {ext }}$ both NTA domains then we say $\Omega$ is a 2-sided NTA domain. Note that in either case $\Omega$ is a UR domain.

Definition 1.18. (Chord arc domain and 2-sided Chord arc domain). We say that a domain $\Omega \subset \mathbb{R}^{n+1}$ is a chord arc (resp. 2-sided chord arc) domain, if $\Omega$ is an NTA (resp. 2-sided NTA) domain, and $\partial \Omega$ is n-dimensional ADR.

Definition 1.19. (Measure theoretic boundary). Given $\Omega \subset \mathbb{R}^{n+1}$, a set of locally finite perimeter ${ }^{3}$, we say that $x \in \partial_{*} \Omega$, the measure theoretic boundary of $\Omega$, if

$$
\limsup _{r \rightarrow 0} \frac{|B(x, r) \cap \Omega|}{r^{n+1}}>0
$$

and

$$
\limsup _{r \rightarrow 0} \frac{\left|B(x, r) \cap \Omega^{c}\right|}{r^{n+1}}>0,
$$

where $|A|$ is the Lebesgue measure of $A$. Given a domain $\Omega \subseteq \mathbb{R}^{n+1}$ we say that the measure theoretic boundary has full measure if $H^{n}\left(\partial \Omega \backslash \partial_{*} \Omega\right)=0$. One should note that if $\Omega \subset \mathbb{R}^{n+1}$ is a set of locally finite perimeter then the measure theoretic boundary and the reduced boundary differ by a set of $H^{n}$ measure zero, so it then follows that the measure theoretic boundary has full measure if and only the reduced boundary has full measure (see [EG, Section 5.8]).

Definition 1.22. (Nontangential approach region and maximal function). Fix $\alpha>0$ and let $\Omega$ be a domain then for $x \in \partial \Omega$ we define the nontangential approach region (or "cone")

$$
\Gamma(x)=\Gamma_{\alpha}(x)=\{Y \in \Omega:|Y-x|<(1+\alpha) \delta(Y)\} .
$$

\footnotetext{
${ }^{3}$ We note that if $\partial \Omega$ is ADR (in particular, if it is UR), then $\Omega$ has locally finite perimeter, by the criterion in [EG, Theorem 1, p. 222].
} 
We also define the nontangential maximal function for $u: \Omega \rightarrow \mathbb{R}$

$$
\mathcal{N} u(x)=\mathcal{N}_{\alpha} u(x)=\sup _{Y \in \Gamma_{\alpha}(x)}|u(Y)|, \quad x \in \partial \Omega .
$$

We make the convention that $\mathcal{N} u(x)=0$ when $\Gamma_{\alpha}(x)=\varnothing$.

Definition 1.25. (VMO and VMO $\mathbf{V O}_{l o c}$ ). Let $E \subset \mathbb{R}^{n+1}$ be $n$-dimensional ADR, and let $\sigma:=\left.H^{n}\right|_{E}$ as above. We denote by $\operatorname{VMO}(d \sigma)=V M O(E, d \sigma)$ the closure of the set of bounded uniformly continuous functions defined on $E$ in $B M O(E, d \sigma)$. We say that $f \in V M O_{l o c}(d \sigma)$ if

$$
\lim _{r \rightarrow 0} \sup _{x \in K} f_{\Delta(x, r)}\left|f(x)-f_{\Delta(x, r)} f\right| d \sigma=0,
$$

for every compact $K \subset E$. Of course, it is well known that (1.26) holds with $E$ in place of $K$ in the supremum, if and only if $f \in V M O(E, d \sigma)$. Thus, $V M O$ and $V M O_{l o c}$ are distinct only for unbounded $E$.

We now record some estimates and some known results that will be important in the proofs of the theorems.

1.2. Preliminary Estimates and Observations. In the sequel, we will sometimes assume more on $\Omega$ and $\Omega_{\text {ext }}$, however $\Omega$ and $\Omega_{\text {ext }}$ will always be UR domains. In addition, $k_{1}$ will be the Poisson kernel for the domain $\Omega$ and $k_{2}$ will be the Poisson kernel for $\Omega_{\text {ext }}$.

Suppose that $\log k_{1}^{X_{1}} \in V M O_{l o c}(d \sigma)$. Let $\omega=\omega^{X_{1}}$ and $k=k^{X_{1}}$ be the harmonic measure and Poisson Kernel for $\Omega$ with pole at $X_{1}$ (resp.). Then by the definition of $V M O_{l o c}(d \sigma)$, for each $B_{0}:=B\left(x_{0}, r_{0}\right)$, with $x_{0} \in \partial \Omega$, and $r_{0}>0$, and for each $\eta>0$, there exists $r_{1}>0$ such that if $x \in \partial \Omega \cap B_{0}$, and $s \in\left(0, r_{1}\right)$, then

$$
f_{\Delta(x, s)}\left|\log k(z)-f_{\Delta(x, s)} \log k(y) d \sigma(y)\right| d \sigma(z) \leq \eta \text {. }
$$

Since $\partial \Omega$ is ADR, it follows that $k$ and $k^{-1}$ belong to $R H_{q, l o c}(d \sigma)$ ("local Reverse Hölder- $q$ "), for every $q<\infty$; i.e., given $B_{0}=B\left(x_{0}, r_{0}\right)$, for every $q \in(1, \infty)$, there exists $C_{q, B_{0}}$ such that for any surface ball $\Delta=\Delta(x, r)$, with $x \in B_{0} \cap \partial \Omega$, and $r \leq r_{0}$,

$$
\left(f_{\Delta} k^{q} d \sigma\right)^{1 / q} \leq C_{q, B_{0}} f_{\Delta} k d \sigma
$$

and

$$
\left(f_{\Delta} k^{-q} d \sigma\right)^{1 / q} \leq C_{q, B_{0}} f_{\Delta} k^{-1} d \sigma
$$

See, e.g., [KT3, Theorem 2.1, p. 332], or the references cited there. As a consequence of (1.28) and (1.29) (holding for all $q<\infty$ ) we have the following Lemma.

Lemma 1.30. ([KT3] Corollary 2.4) Suppose that $\log k \in V M O_{l o c}(d \sigma)$. Fix $B_{0}:=$ $B\left(x_{0}, r_{0}\right)$, with $x_{0} \in \partial \Omega$ and $r_{0}>0$. Then for all $\beta>0, w \in B_{0} \cap \partial \Omega, s \leq r_{0}$, and $E \subset \Delta(w, s)=: \Delta$,

$$
C_{\beta, B_{0}}^{-1}\left(\frac{\sigma(E)}{\sigma(\Delta)}\right)^{1+\beta} \leq \frac{\omega(E)}{\omega(\Delta)} \leq C_{\beta, B_{0}}\left(\frac{\sigma(E)}{\sigma(\Delta)}\right)^{1-\beta} .
$$


The reverse Hölder estimate (1.28) for some fixed $q>1$ (i.e., the $A_{\infty}$ property) yields an exponential reverse Jensen inequality, so that for any $\Delta$ as in (1.28),

$$
e^{f_{\Delta} \log k d \sigma} \approx f_{\Delta} k d \sigma=\frac{\omega(\Delta)}{\sigma(\Delta)},
$$

with implicit constants that may depend on $B_{0}$. See [GR, Theorem 2.15, p. 405] for a proof of (1.32). For the connection between $A_{\infty}$ and BMO, see [GR, Corollary 2.19 , p. 409].

We shall require also the following.

Lemma 1.33. Let $B_{0}:=B\left(x_{0}, r_{0}\right)$, with $x_{0} \in \partial \Omega$ and $r_{0}>0$. Given $\epsilon \in(0,1)$, let $r_{1}>0$ be such that (1.27) holds for all $x \in \partial \Omega \cap B_{0}$ and $s \in\left(0, r_{1}\right)$, with $\eta=\epsilon$. Let $p \in(1, \infty)$ and suppose that $M r \in\left(0, r_{1}\right)$. If we set $\Delta^{*}:=\Delta(x, M r)$, and $a:=a_{x, M r}=e^{f_{\Delta^{*}} \log k d \sigma}$, one then has

$$
\left(f_{\Delta^{*}}\left|1-\frac{k}{a}\right|^{p} d \sigma\right)^{1 / p} \leq C_{1} \epsilon^{b}
$$

with $b=b(p)=(4 p)^{-1}$, and for some $C_{1}=C_{1}\left(p, B_{0}\right)$, uniformly for $x \in \partial \Omega \cap B_{0}$ and $r<r_{1} / M$.

Remark. Applying (1.32) with $\Delta^{*}$ in place of $\Delta$, we note for future reference that

$$
a \approx \frac{\omega(\Delta(x, M r))}{\sigma(\Delta(x, M r))},
$$

Proof. Set $A:=\left\{z \in \Delta^{*}:\left|\log k(z)-f_{\Delta^{*}} \log k(y) d \sigma(y)\right|>\sqrt{\epsilon}\right\}$, so that by Chebyshev's inequality one has

$$
\sigma(A) \leq \sqrt{\epsilon} \sigma\left(\Delta^{*}\right) .
$$

Set $F:=\Delta^{*} \backslash A$. Then by definition of $a$, we have for $z \in F$,

$$
|\log k(z)-\log a| \leq \sqrt{\epsilon} .
$$

Exponentiating (1.37) we obtain

$$
e^{-\sqrt{\epsilon}} \leq \frac{k(z)}{a} \leq e^{\sqrt{\epsilon}},
$$

so that by Taylor's Theorem one has

$$
\left|1-\frac{k(z)}{a}\right| \leq C \sqrt{\epsilon}, \quad \forall z \in F .
$$

Then we have

$$
\begin{aligned}
\left(f_{\Delta^{*}}\left|1-\frac{k}{a}\right|^{p} d \sigma\right)^{1 / p} & \\
\leq\left(\frac{1}{\sigma\left(\Delta^{*}\right)} \int_{F}\left|1-\frac{k}{a}\right|^{p} d \sigma\right)^{1 / p} & +\left(\frac{1}{\sigma\left(\Delta^{*}\right)} \int_{A}\left|1-\frac{k}{a}\right|^{p} d \sigma\right)^{1 / p} \\
& \lesssim \sqrt{\epsilon}+\left(\frac{1}{\sigma\left(\Delta^{*}\right)} \int_{A}\left|1-\frac{k}{a}\right|^{p} d \sigma\right)^{1 / p},
\end{aligned}
$$


Using Minkowski's inequality, (1.36), (1.35), and that $k \in R H_{2 p}$ one has

$$
\begin{aligned}
\left(\frac{1}{\sigma\left(\Delta^{*}\right)} \int_{A}\left|1-\frac{k}{a}\right|^{p} d \sigma\right)^{1 / p} & \leq\left(\frac{\sigma(A)}{\sigma\left(\Delta^{*}\right)}\right)^{1 / p}+\frac{1}{a}\left(\frac{1}{\sigma\left(\Delta^{*}\right)} \int_{A} k^{p}\right)^{1 / p} \\
& \leq \epsilon^{\frac{1}{2 p}}+\left(\frac{\sigma(A)}{\sigma\left(\Delta^{*}\right)}\right)^{\frac{1}{2 p}} \frac{1}{a}\left(\frac{1}{\sigma\left(\Delta^{*}\right)} \int_{A} k^{2 p} d \sigma\right)^{1 / 2 p} \\
& \leq \epsilon^{\frac{1}{2 p}}+\epsilon^{\frac{1}{4 p}} \frac{1}{a} f_{\Delta^{*}} k d \sigma \lesssim \epsilon^{\frac{1}{4 p}},
\end{aligned}
$$

where the implicit constants may depend on $B_{0}$. Then, since $p>1$, putting (1.40) and (1.41) together we obtain (1.34), with $C_{1}$ depending on $B_{0}$ and $p$.

Since $\partial \Omega$ is UR, for all $p \in(1, \infty)$ we have

$$
\|\mathcal{N}(\nabla \mathcal{S} f)\|_{p} \leq C\|f\|_{p},
$$

where $C$ depends on the UR character of $\partial \Omega$, dimension, $p$, and the aperture of the cones defining $\mathcal{N}$. Estimate (1.42) is essentially proved in [DS1] (bounds for the non-tangential maximal function of $\nabla \mathcal{S} f$ follow from uniform bounds for the truncated singular integrals, plus a standard Cotlar Lemma argument; the details may be found in [HMT, Proposition 3.20].)

In addition, we have the following result proved in [HMT].

Lemma 1.43. ([HMT, Proposition 3.30]), If $\Omega$ is a UR domain (recall Definition 1.14), then for a.e. $x \in \partial \Omega$, and for all $f \in L^{p}(d \sigma), 1<p<\infty$,

$$
\lim _{\substack{Z \rightarrow x \\ Z \in \Gamma^{-}(x)}} \nabla \mathcal{S} f(Z)=-\frac{1}{2} v(x) f(x)+T f(x),
$$

and

$$
\lim _{\substack{Z \rightarrow x \\ Z \in \Gamma^{+}(x)}} \nabla \mathcal{S} f(Z)=\frac{1}{2} v(x) f(x)+T f(x) .
$$

where $T$ is a principal value singular integral operator, $\Gamma^{+}(x)$ is the cone at $x$ relative to $\Omega, \Gamma^{-}(x)$ is the cone at x relative to $\Omega_{\mathrm{ext}}$, and $v$ is the outer normal to $\Omega$.

Remark 1.46. We have taken our fundamental solution to be positive, so for that reason there are some changes in sign in both (1.44) and (1.45) as compared to the formulation in [HMT].

Remark 1.47. Recall that by Definition 1.14, we have assumed in particular that $\sigma\left(\partial \Omega \backslash \partial \Omega_{*}\right)=0$, and therefore the measure theoretic outer unit normal $v$ exists a.e. on $\partial \Omega$ (see [EG, Chapter 5]).

We recall now some fundamental estimates relating harmonic measure and the Green function.

Lemma 1.48. ([JK]) Let $\Omega \subset \mathbb{R}^{n+1}$ be an NTA domain and let $G(X, Y)$ denote the Green function of $\Omega$. Let $x \in \partial \Omega, R \in(0$, diam $\partial \Omega)$, and let $Y_{R}$ be a corkscrew point 
for $B(x, R)$. If $X \in \Omega \backslash B(x, 2 R)$, then

$$
G\left(X, Y_{R}\right) \approx \frac{\omega^{X}(\Delta(x, R))}{R^{n-1}} .
$$

Moreover, if $g(Y)$ is the Green function with pole at infinity, and $\omega$ is harmonic measure associated to $g$ (see [KT4]) then

$$
G\left(Y_{R}\right) \approx \frac{\omega(\Delta(x, R))}{R^{n-1}} .
$$

The implicit constants in (1.49)-(1.50) depend only on dimension and the NTA constants.

Remark. In the case that the pole is finite (estimate (1.49)), this result was proved in $[\mathrm{JK}]$; the result for pole at infinity follows from the finite case, plus the construction in [KT2]. Estimate (1.49) has been extended to general divergence form elliptic operators in [CFMS].

Lemma 1.51. ([JK]) Let $\Omega$ be an NTA domain and suppose that $u$ is harmonic in $\Omega$ and vanishes continuously on $\Delta(x, 2 r)$ then

$$
\sup _{X \in B(x, r) \cap \Omega} u(X) \lesssim u\left(Y_{x, r}\right)
$$

where $Y_{x, r}$ is a corkscrew point for $\Delta(x, r)$. The implicit constants depend only on the NTA constants and dimension.

We also recall a result proved by Bourgain about harmonic measure on domains with ADR boundary.

Lemma 1.53. ([Bo]) Let $\Omega \subset \mathbb{R}^{n+1}$ be an open set with $n$-dimensional ADR boundary $\partial \Omega$. Then there exists constants $C_{0}>2$, and $c_{1}>0$, depending on dimension and $A D R$ such that for all $x_{1} \in \partial \Omega$ and $R \in(0, \operatorname{diam}(\partial \Omega))$, if $Y \in \Omega \cap B\left(x_{1}, R\right)$ then

$$
\omega^{Y}\left(B\left(x_{1}, C_{0} R\right)\right) \geq c_{1} .
$$

\section{Proofs Theorems 1.1 AND 1.2}

Proof of Theorem 1.1. Fix $B_{0}=B\left(x_{0}, r_{0}\right)$, and $B_{0}^{\star}:=B\left(x_{0}, r_{0}^{\star}\right)$, with $x_{0} \in \partial \Omega$, $r_{0}>0$, and $r_{0}^{\star}=100 C_{0}\left(\left|X_{1}-x_{0}\right|+r_{0}+1\right)$, where $C_{0}$ is the constant in Lemma 1.53. Fix also $\epsilon \in(0,1)$, and let $r_{1}>0$ be such that (1.27) holds with $\eta=\epsilon$, for $x \in$ $\partial \Omega \cap B_{0}^{\star}$ and $s \in\left(0, r_{1}\right)$. Without loss of generality we take $r_{1}<\min \left\{\frac{\delta\left(X_{1}\right)}{50}, 1, r_{0}\right\}$. Let $M:=50 \epsilon^{-\frac{1}{8 n}}$, and suppose that $M r \in\left(0, r_{1}\right)$. We now fix $x \in B_{0} \cap \partial \Omega$, and for any points $y, z \in \Delta(x, r)$, let $y^{*}, z^{*}$ denote arbitrary points in $\Gamma^{-}(y) \cap B(y, r / 2)$ and in $\Gamma^{-}(z) \cap B(z, r / 2)$, respectively. Setting

$$
\Delta:=\Delta(x, r), \quad \Delta^{*}:=\Delta(x, M r),
$$

we shall first prove that for any such $y, z, y^{*}, z^{*}$,

$$
\left(f_{\Delta}\left|\nabla \mathcal{S} 1_{\Delta^{*}}\left(z^{*}\right)-f_{\Delta} \nabla \mathcal{S} 1_{\Delta^{*}}\left(y^{*}\right) d \sigma(y)\right|^{2} d \sigma(z)\right)^{\frac{1}{2}} \leq C \epsilon^{\gamma},
$$


where $C=C\left(n, U R, B_{0}, B_{0}^{\star}, \delta\left(X_{1}\right),\left|X_{1}-x_{0}\right|\right)$, and where $\gamma$ is a positive constant, depending only on dimension. For the sake of notational convenience, in the sequel, we shall often allow generic and implicit constants to depend upon these parameters, without explicitly making note of such dependence. As before, set $a:=a_{x, M r}=e^{f_{\Delta^{*}} \log k d \sigma}$ and write

$$
1_{\Delta^{*}}=\left[\left(1-\frac{k}{a}\right) 1_{\Delta^{*}}\right]+\left[\frac{k}{a}\right]-\left[\left(\frac{k}{a}\right) 1_{(\Delta *)^{c}}\right] .
$$

Using (2.2) we have that the left hand side of (2.1) is bounded by the sum of three terms $I, I I$ and $I I I$ where

$$
\begin{aligned}
& I=\left(f_{\Delta}\left|\nabla \mathcal{S}\left[\left(1-\frac{k}{a}\right) 1_{\Delta^{*}}\right]\left(z^{*}\right)-f_{\Delta} \nabla \mathcal{S}\left[\left(1-\frac{k}{a}\right) 1_{\Delta^{*}}\right]\left(y^{*}\right) d \sigma(y)\right|^{2} d \sigma(z)\right)^{\frac{1}{2}}, \\
& \text { (2.4) } \quad I I=\left(f_{\Delta}\left|\nabla \mathcal{S}\left[\frac{k}{a}\right]\left(z^{*}\right)-f_{\Delta} \nabla \mathcal{S}\left[\frac{k}{a}\right]\left(y^{*}\right) d \sigma(y)\right|^{2} d \sigma(z)\right)^{\frac{1}{2}},
\end{aligned}
$$

and

$$
I I I=\left(f_{\Delta}\left|\nabla \mathcal{S}\left[\left(\frac{k}{a}\right) 1_{(\Delta *)^{c}}\right]\left(z^{*}\right)-f_{\Delta} \nabla \mathcal{S}\left[\left(\frac{k}{a}\right) 1_{(\Delta *)^{c}}\right]\left(y^{*}\right) d \sigma(y)\right|^{2} d \sigma(z)\right)^{\frac{1}{2}} .
$$

We begin by estimating $I$. By (1.42) and Lemma 1.33 with $p=2$, we have

$$
\begin{aligned}
I \leq 2\left(f_{\Delta}\left|\mathcal{N}\left(\nabla \mathcal{S}\left[\left(1-\frac{k}{a}\right) 1_{\Delta^{*}}\right]\right)\right|^{2} d \sigma\right)^{\frac{1}{2}} \\
\qquad M^{\frac{n}{2}}\left(f_{\Delta^{*}}\left|1-\frac{k}{a}\right|^{2} d \sigma\right)^{\frac{1}{2}} \lesssim M^{\frac{n}{2}} \epsilon^{\frac{1}{8}} \lesssim \epsilon^{\frac{1}{16}} .
\end{aligned}
$$

Now for $I I$, we recall that $k=k_{1}^{X_{1}}$ is harmonic measure for $\Omega$ with pole at $X_{1}$. Moreover, $\mathcal{E}\left(\cdot-z^{*}\right)$ and $\mathcal{E}\left(\cdot-y^{*}\right)$ are harmonic in $\Omega$ since $z^{*}, y^{*} \in \Omega_{\text {ext }}$, and decay to 0 at infinity, and are therefore equal to their respective Poisson integrals in $\Omega$. Consequently,

$$
I I=\frac{1}{a}\left(f_{\Delta} f_{\Delta}\left|\nabla \mathcal{E}\left(X_{1}-z^{*}\right)-\nabla \mathcal{E}\left(X_{1}-y^{*}\right) d \sigma(y)\right|^{2} d \sigma(z)\right)^{\frac{1}{2}} .
$$

Recall that by definition, $M r+r_{0}<2 r_{0} \ll r_{0}^{\star}$, and that $x \in B_{0} \cap \partial \Omega$, hence $B(x, M r) \subset 2 B_{0} \subset B_{0}^{\star}$. Thus, we may apply Lemma 1.30, with $r_{0}^{\star}, B_{0}^{\star}$ in place of $r_{0}, B_{0}$, with $w=x_{0}$ and $s=r_{0}^{\star}$ (thus, $B(w, s)=B_{0}^{\star}, \Delta=\Delta_{0}^{\star}=B_{0}^{\star} \cap \partial \Omega$ ), and with $E=\Delta(x, M r)$, to deduce that

$$
\frac{\omega\left(\Delta_{0}^{\star}\right)}{\omega(\Delta(x, M r)} \lesssim\left(\frac{r_{0}^{\star}}{M r}\right)^{n+\tau}, \quad \forall \tau>0,
$$

where the implicit constant of course depends upon $\tau$ and $B_{0}^{\star}$. 
We note that by Lemma 1.53, and the definition of $r_{0}^{\star}$, there is a uniform constant $c>0$ such that

$$
\omega\left(\Delta_{0}^{\star}\right) \geq c .
$$

We further note that, since $y^{*}, z^{*} \in B(x, 2 r)$,

$$
\left|\nabla \mathcal{E}\left(X_{1}-z^{*}\right)-\nabla \mathcal{E}\left(X_{1}-y^{*}\right)\right| \lesssim \frac{r}{\delta\left(X_{1}\right)^{n+1}} .
$$

Then continuing (2.7), we have, using (1.35), (2.8) (with $\tau=1 / 2$ ), and (2.9),

$$
\begin{aligned}
I I \lesssim \frac{1}{a} \frac{r}{\delta\left(X_{1}\right)^{n+1}} & \approx \frac{\sigma(\Delta(x, M r))}{\omega(\Delta(x, M r))} \frac{r}{\delta\left(X_{1}\right)^{n+1}} \\
= & \frac{\sigma(\Delta(x, M r))}{\omega\left(\Delta_{0}^{\star}\right)} \frac{\omega\left(\Delta_{0}^{\star}\right)}{\omega(\Delta(x, M r))} \frac{r}{\delta\left(X_{1}\right)^{n+1}} \\
& \lesssim(M r)^{n}\left(\frac{r_{0}^{\star}}{M r}\right)^{n+\frac{1}{2}} \frac{r}{\delta\left(X_{1}\right)^{n+1}} \lesssim M^{-\frac{1}{2}} r^{\frac{1}{2}} \lesssim \frac{1}{M} \approx \epsilon^{\frac{1}{8 n}},
\end{aligned}
$$

since $r<r_{1} / M \ll \delta\left(X_{1}\right) / M$, where we remind the reader that in this part of the argument, we allow implicit constants to depend upon $\delta\left(X_{1}\right)$, and on the various parameters involved in the definition of $r_{0}^{\star}$.

For III we use basic Calderón-Zygmund type estimates as follows. Let

$$
\Delta_{j}^{\prime}:=\Delta\left(x, 2^{j} r\right), \quad A_{j}^{\prime}:=\Delta_{j}^{\prime} \backslash \Delta_{j-1}^{\prime},
$$

so that

(2.11) $\quad I I I=$

$$
\begin{gathered}
\left(f_{\Delta}\left|f_{\Delta}\left(\nabla \mathcal{S}\left[\left(\frac{k}{a}\right) 1_{(\Delta *)^{c}}\right]\left(z^{*}\right)-\nabla \mathcal{S}\left[\left(\frac{k}{a}\right) 1_{(\Delta *)^{c}}\right]\left(y^{*}\right)\right) d \sigma(y)\right|^{2} d \sigma(z)\right)^{\frac{1}{2}} \\
=\left(f_{\Delta}\left|f \int_{\Delta}\left[\nabla \mathcal{E}\left(z^{*}-w\right)-\nabla \mathcal{E}\left(y^{*}-w\right)\right] \frac{k(w)}{a} d \sigma(w) d \sigma(y)\right|^{2} d \sigma(z)\right)^{\frac{1}{2}} \\
\leq \sum_{j: 2^{j} \geq M}\left(f_{\Delta}\left[f_{\Delta} \int_{A_{j}^{\prime}}\left|\nabla \mathcal{E}\left(z^{*}-w\right)-\nabla \mathcal{E}\left(y^{*}-w\right)\right| \frac{k(w)}{a} d \sigma(w) d \sigma(y)\right]^{2} d \sigma(z)\right)^{\frac{1}{2}} \\
\lesssim \sum_{j: 22^{\prime} \geq M}\left(f_{\Delta}\left[f_{\Delta} \int_{A_{j}^{\prime}} \frac{r}{\left(2^{j} r\right)^{n+1}} \frac{k(w)}{a} d \sigma(w) d \sigma(y)\right]^{2} d \sigma(z)\right)^{\frac{1}{2}} \\
=\sum_{j \in J_{1}} \ldots+\sum_{j \in J_{2}} \ldots=: I I I_{1}+I I I_{2},
\end{gathered}
$$

where

$$
J_{1}:=\left\{j: M \leq 2^{j} \leq r_{0}^{\star} / r\right\}, \quad J_{2}:=\left\{j: 2^{j}>r_{0}^{\star} / r\right\} .
$$


For $j \in J_{1}$, we may apply Lemma 1.30 , with $B_{0}^{\star}$ in place of $B_{0}$, and with $\Delta=\Delta_{j}^{\prime}$ and $E=\Delta(x, M r)$, to obtain

$$
\frac{\omega\left(\Delta_{j}^{\prime}\right)}{\omega(\Delta(x, M r)} \lesssim\left(\frac{2^{j}}{M}\right)^{n+\beta n}
$$

We then have

$$
\begin{aligned}
I I I_{1} \lesssim \sum_{j \in J_{1}} & \frac{1}{2^{j}}\left(f_{\Delta}\left[f_{\Delta} f_{\Delta_{j}^{\prime}} \frac{k(w)}{a} d \sigma(w) d \sigma(y)\right]^{2} d \sigma(z)\right)^{\frac{1}{2}} \\
\lesssim \sum_{j \in J_{1}} \frac{1}{2^{j}} \frac{1}{a} \frac{\omega\left(\Delta_{j}^{\prime}\right)}{\sigma\left(\Delta_{j}^{\prime}\right)} & \approx \sum_{j \in J_{1}} \frac{1}{2^{j}} \frac{\sigma(\Delta(x, M r))}{\sigma\left(\Delta_{j}^{\prime}\right)} \frac{\omega\left(\Delta_{j}^{\prime}\right)}{\omega(\Delta(x, M r)} \\
& \lesssim \sum_{j: 2^{j} \geq M} 2^{-j}\left(\frac{M}{2^{j}}\right)^{n}\left(\frac{2^{j}}{M}\right)^{n+1 / 2} \lesssim \frac{1}{M} \lesssim \epsilon^{\frac{1}{8 n}},
\end{aligned}
$$

where in the middle line we have used (1.35), and in the last line, the ADR property and (2.12) with $\beta=1 /(2 n)$. Since $\omega$ is a probablility measure, we also have that

$$
\begin{aligned}
I I I_{2} \lesssim \sum_{j: 2^{j} r r_{0}^{\star}} r\left(2^{j} r\right)^{-n-1} a^{-1} \approx r\left(\frac{1}{r_{0}^{\star}}\right)^{n+1} \frac{\sigma(\Delta(x, M r))}{\omega(\Delta(x, M r))} \\
=r\left(\frac{1}{r_{0}^{\star}}\right)^{n+1} \frac{\sigma(\Delta(x, M r))}{\omega\left(\Delta_{0}^{*}\right)} \frac{\omega\left(\Delta_{0}^{*}\right)}{\omega(\Delta(x, M r))} \lesssim r\left(\frac{1}{r_{0}^{\star}}\right)^{n+1}(M r)^{n}\left(\frac{r_{0}^{\star}}{M r}\right)^{n+1 / 2} \\
=\left(\frac{r}{r_{0}^{\star}}\right)^{1 / 2} M^{-1 / 2} \lesssim M^{-1} \approx \epsilon^{\frac{1}{8 n}},
\end{aligned}
$$

where in the middle line we have used the ADR property, (2.9), and (2.8) (with $\tau=1 / 2$ ), and in the last line that $M r \leq r_{1} \ll r_{0}^{\star}$. Combining the estimates for $I$, $I I, I I I_{1}$ and $I I_{2}$, we obtain (2.1) with $\gamma=1 /(8 n)$ (or with $\gamma=1 / 16$, if $n=1$ ).

With (2.1) in hand, we continue with the proof of Theorem 1.1. Setting

$$
\text { n.t. } \nabla \mathcal{S}^{-} f(x):=\lim _{\substack{Z \rightarrow x \\ Z \in \Gamma^{-}(x)}} \nabla \mathcal{S} f(Z),
$$

since the limit exists for a.e. $x \in \partial \Omega$ (see Lemma 1.43), we may now use (1.42), (2.1), and dominated convergence to obtain

$$
\left(f_{\Delta} \mid n . t . \nabla \mathcal{S}^{-} 1_{\Delta^{*}}(z)-f_{\Delta} \text { n.t. }\left.\nabla \mathcal{S}^{-} 1_{\Delta^{*}}(y) d \sigma(y)\right|^{2} d \sigma(z)\right)^{\frac{1}{2}} \leq C \epsilon^{\gamma},
$$

for $x \in \partial \Omega \cap B_{0}$ and $r<r_{1} / M$. In addition since $\log k_{2}^{X_{2}} \in V M O(d \sigma)$ the same analysis shows that (2.13) holds for $n . t . \nabla \mathcal{S}^{-} 1_{\Delta^{*}}$ replaced with

$$
\text { n.t. } \nabla \mathcal{S}^{+} 1_{\Delta^{*}}:=\lim _{\substack{\left.Z \rightarrow \Gamma^{+} \\ Z \in \Gamma^{(} x\right)}} \nabla \mathcal{S} 1_{\Delta^{*}}(Z) .
$$

By (1.44) and (1.45)

$$
v(x) 1_{\Delta^{*}}(x)=\lim _{\substack{Z \rightarrow x \\ Z \in \Gamma^{+}(x)}} \nabla \mathcal{S} 1_{\Delta^{*}}(Z)-\lim _{\substack{Z \rightarrow x \\ Z \in \Gamma^{-}(x)}} \nabla \mathcal{S} 1_{\Delta^{*}}(Z) .
$$


Thus, since $\Delta \subset \Delta^{*}$, by (2.13) and its analogue for $\mathcal{S}^{+}$, we obtain

$$
\left(f_{\Delta}\left|v(z)-f_{\Delta} v(y) d \sigma(y)\right|^{2} d \sigma(z)\right)^{\frac{1}{2}} \leq C \epsilon^{\gamma}
$$

for $x \in \partial \Omega \cap B_{0}$ and $0<r<M^{-1} r_{1}(\epsilon) \approx \epsilon^{1 /(8 n)} r_{1}(\epsilon)$. Hence, $v \in V M O_{l o c}(d \sigma)$.

Proof of Theorem 1.2. The proof is nearly the same as that of Theorem 1.1, with a few minor differences. Recall that in contrast to the situation for Theorem 1.1, we now impose the stronger assumption that $\Omega$ is a chord arc domain and that $\log k_{1} \in V M O$ (globally). In this setting, Lemma 1.48 and Lemma 1.51 hold, and harmonic measure is doubling. Moreover, (1.27), Lemma 1.30 and Lemma 1.33 hold globally (i.e., not localized to a ball $B_{0}$ ). Following the proof of Theorem 1.1, we proceed as follows.

As before, we fix $\epsilon \in(0,1)$, and let $r_{1}>0$ be such that (1.27) holds with $\eta=\epsilon$, for $x \in \partial \Omega$ and $s \in\left(0, r_{1}\right)$. We take $r_{1}<\min \left\{\frac{\delta\left(X_{1}\right)}{50}, 1\right\}$, let $M:=50 \epsilon^{-\frac{1}{8 n}}$, and suppose that $M r \in\left(0, r_{1}\right)$. Given $x \in \partial \Omega$, we set $\Delta:=\Delta(x, r)$ and $\Delta^{*}:=\Delta(x, M r)$, and for $y, z \in \Delta$, we let $y^{*}, z^{*}$ denote arbitrary points in $\Gamma^{-}(y) \cap B(y, r / 2)$, and $\Gamma^{-}(z) \cap B(z, r / 2)$, respectively. Once again, we seek to prove (2.1), but now with $C=C(n, U R)$.

We break up $1_{\Delta^{*}}$ as in (2.2) and estimate the left hand side of (2.1) by the same three terms, $I, I I$ and $I I I$. We estimate term $I$ exactly as before.

Next, we will show that

$$
I I=\left(f_{\Delta}\left|\nabla \mathcal{S}\left[\frac{k}{a}\right]\left(z^{*}\right)-f_{\Delta} \nabla \mathcal{S}\left[\frac{k}{a}\right]\left(y^{*}\right) d \sigma(y)\right|^{2} d \sigma(z)\right)^{\frac{1}{2}}=0,
$$

for $k=k_{1}$, the Poisson kernel at infinity. Fix $x_{0} \in \partial \Omega$ and $R \gg r$, and let $\varphi_{R}(X) \in$ $C_{0}^{\infty}$ be a postivite smooth cutoff function on $B\left(x_{0}, R\right)$ such that $\operatorname{supp} \varphi \subset B\left(x_{0}, R\right)$, $|\nabla \varphi| \lesssim \frac{1}{R},\left|\nabla^{2} \varphi\right| \lesssim \frac{1}{R^{2}}$, and $\varphi \equiv 1$ on $B\left(x_{0}, \frac{R}{2}\right)$. Let

$$
\begin{aligned}
& I I_{R}:= \\
& \left(f_{\Delta}\left|f_{\Delta} \int_{\partial \Omega}\left[\nabla \mathcal{E}\left(w-z^{*}\right)-\nabla \mathcal{E}\left(w-y^{*}\right)\right] \varphi_{R}(w)\left[\frac{k(w)}{a}\right] d \sigma(w) d \sigma(y)\right|^{2} d \sigma(z)\right)^{\frac{1}{2}}
\end{aligned}
$$

If we set $A_{R}=B\left(x_{0}, R\right) \backslash B\left(x_{0}, \frac{R}{2}\right)$, let $g(X)$ be the Green function with pole at infinity, and let $\mathcal{L}:=\nabla \cdot \nabla$ denote the usual Laplacian in $\mathbb{R}^{n+1}$, then by definition

$$
\begin{aligned}
& I I_{R}^{2}= \\
& \frac{1}{a^{2}} f_{\Delta}\left|f_{\Delta} \iint_{\Omega} \mathcal{L}\left(\left[\nabla \mathcal{E}\left(W-z^{*}\right)-\nabla \mathcal{E}\left(W-y^{*}\right)\right] \varphi_{R}(W)\right) g(W) d W d \sigma(y)\right|^{2} d \sigma(z) \\
& \quad \lesssim \frac{1}{a^{2}}\left(\iint_{A_{R}} g(W) \frac{1}{R^{n+2}} \frac{r}{R} d W\right)^{2}
\end{aligned}
$$


Let $Y_{R}$ be a corkscrew point for $B\left(x_{0}, R\right)$, and set $\Delta_{R}=\partial \Omega \cap B\left(x_{0}, R\right)$. Then using Lemma 1.51, Lemma 1.48 and (1.31) (without dependence on $B_{0}$ ) we have that

$$
\begin{aligned}
\frac{1}{a^{2}}\left(\iint_{A_{R}} g(W) \frac{1}{R^{n+2}} \frac{r}{R} d W\right)^{2} & \lesssim \frac{1}{a^{2}}\left(\frac{r}{R^{2}} \frac{1}{R^{n+1}} \iint_{B\left(x_{0}, R\right)} g(W) d W\right)^{2} \\
& \lesssim \frac{1}{a^{2}}\left(\frac{r}{R^{2}} g\left(Y_{R}\right)\right)^{2} \\
& \lesssim \frac{1}{a^{2}}\left(\frac{r}{R} \frac{\omega\left(\Delta_{R}\right)}{\sigma\left(\Delta_{R}\right)}\right)^{2} \\
& \lesssim\left(\frac{\sigma\left(\Delta\left(x_{0}, M r\right)\right)}{\sigma\left(\Delta_{R}\right)} \frac{\omega\left(\Delta_{R}\right)}{\omega\left(\Delta\left(x_{0}, M r\right)\right)}\right)^{2} \frac{r^{2}}{R^{2}} \\
& \lesssim \frac{r}{R},
\end{aligned}
$$

Thus, for $z^{*}, y^{*}$ as above, $I I_{R} \rightarrow 0$ as $R \rightarrow \infty$, uniformly in the points $z^{*}$ and $y^{*}$ under consideration. By the Dominated Convergence Theorem, we see that

$$
I I=\lim _{R \rightarrow \infty} I I_{R}=0 .
$$

Finally, to handle term $I I I$, we proceed as before until, as in (2.11), we obtain

$$
I I I \lesssim \sum_{j: 2^{j} \geq M}\left(f_{\Delta}\left[f_{\Delta} \int_{A_{j}^{\prime}} \frac{r}{\left(2^{j} r\right)^{n+1}} \frac{k(w)}{a} d \sigma(w) d \sigma(y)\right]^{2} d \sigma(z)\right)^{\frac{1}{2}} .
$$

In the present setting, we can estimate everything as we did for $I I I_{1}$ in the proof of Theorem 1.1 (since we have no dependence on $B_{0}^{\star}$ or $B_{0}$ ). Exactly as before, we find that

$$
I I I \lesssim \epsilon^{\frac{1}{8 n}},
$$

and the conclusion of Theorem 1.2 follows.

Acknowledgements. We are grateful to Chema Martell for pointing out an error in the original version of this manuscript, which could be fixed by working with local VMO spaces.

\section{REFERENCES}

[AC] H. W. Alt and L. A. Caffarelli, Existence and regularity for a minimum problem with free boundary, J. Reine Agnew. Math. 325 (1981) 105-144 1, 2, 3

[Bo] J. Bourgain, On the Hausdorff dimension of harmonic measure in higher dimensions, Invent. Math. 87 (1987), 477483.9

[CFMS] Caffarelli, L.; Fabes, E.; Mortola, S.; Salsa, S. Boundary behavior of nonnegative solutions of elliptic operators in divergence form. Indiana Univ. Math. J. 30 (1981), no. 4, 621-640. 9

[DJ] G. David and D. Jerison, Lipschitz approximation to hypersurfaces, harmonic measure, and singular integrals, Indiana Univ. Math. J. 39 (1990), no. 3, 831-845. 2

[DS1] G. David and S. Semmes, Singular integrals and rectifiable sets in $\mathbb{R}^{n}$ : Beyond Lipschitz graphs, Asterisque 193 (1991). 4, 8

[DS2] G. David and S. Semmes, Analysis of and on Uniformly Rectifiable Sets, Mathematical Monographs and Surveys 38, AMS 1993. 4 
[EG] L.C. Evans and R.F. Gariepy, Measure Theory and Fine Properties of Functions, Studies in Advanced Mathematics, CRC Press, Boca Raton, FL, 1992. 5, 8

[GR] J. García-Cuerva; J. L.Rubio de Francia Weighted norm inequalities and related topics, North-Holland Mathematics Studies, 116. North-Holland Publishing Co., Amsterdam, 1985. 7

[HMT] S. Hofmann, M. Mitrea and M. Taylor, Singular integrals and elliptic boundary problems on regular Semmes-Kenig-Toro domains, Int. Math. Res. Not. 2010, no. 14 2567$28652,3,5,8$

[J] D. Jerison, Regularity of the Poisson kernel and free boundary problems, Colloq. Math. 60-61 (1990), 547-567 1, 3

[JK] D. Jerison and C. Kenig, Boundary behavior of harmonic functions in nontangentially accessible domains, Adv. in Math. 46 (1982), no. 1, 80-147. 5, 8, 9

[Kel] O. Kellog, Foundations of Potential Theory, Springer, Berlin, 1929 (reprinted by Dover, N.Y., 1953). 1

[KT1] C. Kenig and T. Toro, Harmonic measure on locally flat domains, Duke Math. J. 87, (1997), 509-551 1,2

[KT2] C. Kenig and T. Toro, Free Boundary Regularity for harmonic measures and Poisson kernels, Ann. Math. 150 (1999), 369-454 9

[KT3] C. Kenig and T. Toro, Poisson kernel characterization of Reifenberg flat chord arc domains, Ann. Sci. Ecole Norm. Sup. (4) 36 (2003), no.3, 323-401 1, 6

[KT4] C. Kenig and T. Toro, Free boundary regularity below the continuous threshold: 2 phase problems, J. Reine Angew Math. 596 (2006), 1-44 1, 2, 3, 9

[KP] O. Kowalski and D. Preiss, Besicovitch-type properties of measures and submanifolds, J. Reine Angew Math. 379 (1987), 115-151. 2

[M] P. Mattila, Geometry of sets and measures in Euclidean spaces. Fractals and rectifiability, Cambridge Studies in Advanced Mathematics 44, Cambridge University Press, Cambridge, 1995. 4

[MMV] P. Mattila, M. Melnikov and J. Verdera, The Cauchy integral, analytic capacity, and uniform rectifiability, Ann. of Math. (2) 144 (1996), no. 1, 127-136. 4

$[\mathrm{NToV}]$ F. Nazarov, X. Tolsa, and A. Volberg, On the uniform rectifiability of ad-regular measures with bounded Riesz transform operator: The case of codimension 1, Acta Math., to appear. 4

Simon Bortz, Department of Mathematics, University of Missouri, Columbia, MO 65211, USA

E-mail address: sabh8f@mail .missouri .edu

Steve Hofmann, Department of Mathematics, University of Missouri, Columbia, MO 65211 , USA

E-mail address: hofmanns@missouri .edu 